\title{
Predicted performance of LTE transmit diversity schemes in tunnel environment
}

\author{
Concepcion Sanchis-Borras ${ }^{1}$, Pierre Laly ${ }^{2}$, Jose Maria Molina-Garcia-Pardo ${ }^{3}$, Martine Lienard ${ }^{2}$ and Pierre Degauque ${ }^{*}$ \\ ${ }^{1}$ Department of Technical Sciences, Universidad Católica San Antonio de Murcia (UCAM), 30107 Murcia,Spain \\ ${ }^{2}$ University Lille1, IEMN/TELICE, 59655 Villeneuve d Ascq cedex, France \\ ${ }^{3}$ Universidad Politécnica de Cartagena, Dept Tecno. Información Comun., 30202 Cartagena, Spain
}

\begin{abstract}
The Long-Term Evolution broadband will become the dominant technology for the next generation of communication systems. Its performances in terms of capacity and error rate are studying for rural and urban environment. However, even if this is a special case, tunnel environment is often encountered. The objective of this paper is to predict the error rate by using software simulating the proposed LTE data processing and by introducing, in this software, channel transfer functions measured in tunnels. Among all possible transmission modes, emphasis is on the transmit diversity schemes. After a brief description of the channel characteristics and of the simulation process, improvement brought by the possible configurations of transmit diversity is studied, the frequency band being around $3.4 \mathrm{GHz}$.
\end{abstract}

\section{Introduction}

The Long-Term Evolution (LTE) technology is widely studying, the objective being to dramatically increase the bit rate but also to decrease the bit error rate, whatever the environment of the mobile [1], [2]. Its predicted performances are studied both for rural and urban areas and deduced either from simulation or experiments. As an example, results in [3] are based on the 3 GPPP multipath fading channel models: pedestrian, vehicular and typical urban models, while in [4] tests have been performed in a dense-urban environment in a frequency band around 2.3 GHz. However, among all possible environments, road or railway tunnels are a specific case.

In case of a railway always remaining underground, as it may often appear for a metro mass transport system, dedicated transmission schemes based on Multiple-Input Multiple-Output (MIMO) technology can be envisaged. In [5] the MIMO capacity is analytically expressed in terms of waveguide modes, assuming a perfect straight rectangular tunnel, while in [6] results of double directional channel measurements are interpreted owing to a ray tracing simulation tool. MIMO performances presented in [7] and [8] are deduced from an experimental and a theoretical approach, respectively.

However, for road transportation system, the basis of the communication scheme must remain the same outside and inside tunnels. Since LTE will become the dominant technology for the next generation of mobile broadband, its performances inside a tunnel must also be evaluated.

In this paper, we will focus on the physical (PHY) layer and more precisely on the physical downlink shared channel (DL-SCH), because it carries user data and is designed for a wide range of data rates. In LTE release 12 [9], 10 downlink transmission modes are proposed, from the single transmit antenna to the 8 layer transmission. Among all these modes, we will study the improvement, compared to the Single-Input SingleOutput (SISO) case, brought by transmit diversity schemes as the Space Frequency Block Coding (SFBC) and the Frequency Switched Transmit Diversity (FSTD). The channel characteristics used as input data in the tool simulating the PHY layer of the LTE link are those measured in a road tunnel for a transmitting frequency around $3.4 \mathrm{GHz}$. We will assume that both the base station, equivalent to an eNodeB in the LTE terminology, and the mobile (User Equipement - UE) are situated inside the tunnel.

A brief presentation of the tunnel geometry and of the main features of the measurement campaign is given in Section 2. Since the narrow band and wide band channel characteristics varies with the distance between the transmitter (Tx) and the receiver ( $\mathrm{Rx})$ and have a strong impact on link performances, results on path loss, correlation between array antenna elements, coherence bandwidth and delay spread are briefly presented in Section 3. Section 4 describes the downlink processing, simulated with Matlab software, and the studied transmission modes. Evaluation and comparison of the performances of these transmission modes, expressed in terms of bit error rate (BER) for a Quadrature Amplitude Modulation (QAM), are given in Section 5.

\section{Configuration of the tunnel and of the antenna arrays}

Measurements have been performed in a 2-lane straight semi-arched tunnel and whose maximum width and 
height are $6.1 \mathrm{~m}$ and $8.6 \mathrm{~m}$, respectively. In the $\mathrm{Rx}$ and Tx transverse planes, virtual arrays were considered by moving step by step a vertically polarized element. The 10 positions of this antenna, $3 \mathrm{~cm}$ apart, are numbered as shown in Fig. 1 representing half of a tunnel cross section. Note that the drawing of the antenna array is not to scale, its length being $27 \mathrm{~cm}$. The same numbering is used both for the Tx and Rx virtual array elements.

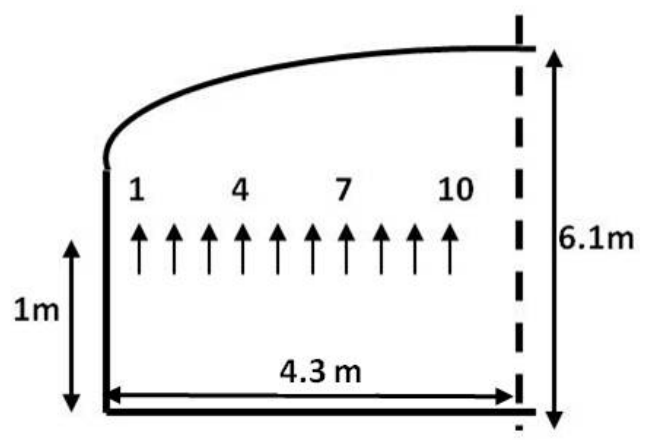

Fig. 1. Half of the tunnel cross-section showing the position of the Tx or Rx antennas and their numbering.

The transfer functions $\mathrm{H}(\mathrm{f})$ between all $\mathrm{Tx}$ and $\mathrm{Rx}$ antenna positions were measured with a network analyzer, connected to the antennas owing to a fiber optics link, and in a wide frequency range extending from 2.8 to $5 \mathrm{GHz}$. Along the tunnel axis, measurement were made with a step of 4 or $6 \mathrm{~m}$. The Tx plane is fixed, the distance $T x-R x$ varying from 50 to $500 \mathrm{~m}$.

The different frequency bands for LTE and LTEadvanced extend from $450 \mathrm{MHz}$ up to $3.8 \mathrm{GHz}$. In the following, we have chosen to simulate a communication link in the upper band, i. e. at $3.4 \mathrm{GHz}$, and considering a useful bandwidth of $5 \mathrm{MHz}$. However, for determining in a first step channel characteristics as coherence bandwidth and delay spread, as defined in [10], results of wide band measurements will be used.

\section{Narrow band and wide band channel characteristics}

The BER presented in Section 4 of course depends on the signal to noise ratio (SNR) along the tunnel. Therefore, we have arbitrarily chosen an average SNR of $8 \mathrm{~dB}$ at the end of the link, i.e. for a distance Tx-Rx between 450 and $500 \mathrm{~m}$. This choice of $8 \mathrm{~dB}$ is justified by the fact that, with such an SNR, the BER is not too small thus allowing a BER calculation with a reasonable time of computation.

Curves in Fig. 2 show the variation of SNR along the tunnel for an Rx antenna at position 1, and a Tx antenna at position 1 or 10 (Fig. 1), these positions being as far apart as possible $(27 \mathrm{~cm}$, i.e. 3.1 wavelength). Since the wavelength is much smaller than the transverse dimensions of the tunnel, average path loss remains of the same order of magnitude whatever the distance but many fading occur, their amplitude and position depending on the position of the Tx antenna in the transverse plane.

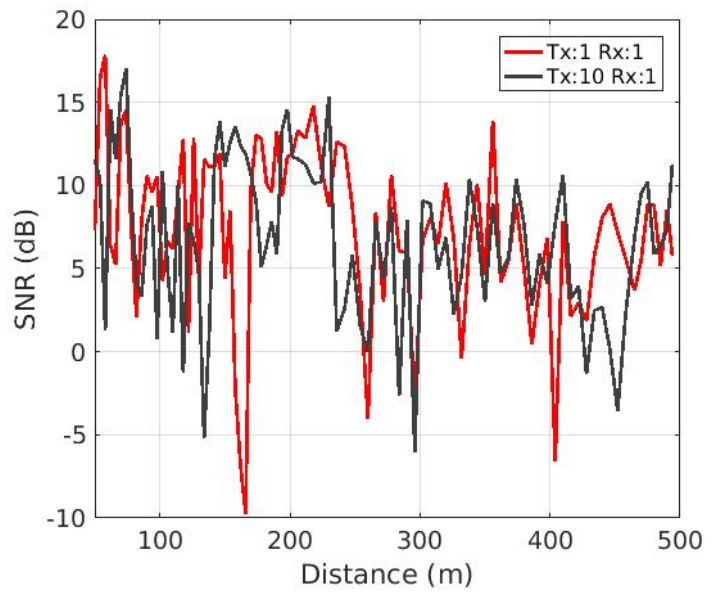

Fig. 2. Variation of the SNR along the tunnel for 2 positions (1 and 10) of the Tx antenna

The fluctuation of the SNR is more important at short distances since the tunnel supports a high number of propagating modes interfering each other, as explained in [5].

The mean correlation coefficient $\rho_{T X}$ between array elements numbered $m$ and $n$ (Fig. 1) at the Tx side, and calculated for 4 distance intervals, are given in Table 1.

Table 1. Mean correlation $\rho_{T X}$ between array elements Tx $m$ and $\mathrm{Tx} n$

\begin{tabular}{|c|c|c|c|c|}
\hline & $50-100 \mathrm{~m}$ & $100-150 \mathrm{~m}$ & $150-200 \mathrm{~m}$ & $200-250 \mathrm{~m}$ \\
\hline Tx1-Tx4 & 0.62 & 0.83 & 0.94 & 0.97 \\
\hline Tx1-Tx7 & 0.51 & 0.63 & 0.86 & 0.90 \\
\hline Tx1-Tx10 & 0.50 & 0.64 & 0.82 & 0.85 \\
\hline
\end{tabular}

$\rho_{T X}$ is of course a decreasing function of the antenna spacing, whatever the distance Tx-Rx. It clearly appears from Table 1 that the correlation takes high values, as soon as the distance becomes larger than $150 \mathrm{~m}$. Indeed, even for a spacing of $27 \mathrm{~cm}$ equivalent to about 3 wavelengths, $\rho_{T X}$ varies from 0.5 at $50 \mathrm{~m}$ to 0.8 beyond a distance of $150 \mathrm{~m}$. Similar results were obtained at the Rx site.

The variation of the coherence bandwidth $\mathrm{Bc}$, calculated for a correlation coefficient of 0.9 , and around a frequency of $3.4 \mathrm{GHz}$, is given in Fig. 3. The smaller values of $\mathrm{Bc}$ occur for short distances due to the high number of interfering modes as previously mentioned, but in any case remains larger than $15 \mathrm{MHz}$.

The channel impulse response was deduced from the transfer function by applying an inverse Fourier transform. Only rays having amplitude attenuated than less of $20 \mathrm{~dB}$, referred to the highest peak, are considered for calculating the delay spread DS. The average value of DS varies between 3 and 6 ns but can reach highest value depending on the position of Rx. In any case, it always remains smaller than $20 \mathrm{~ns}$. 


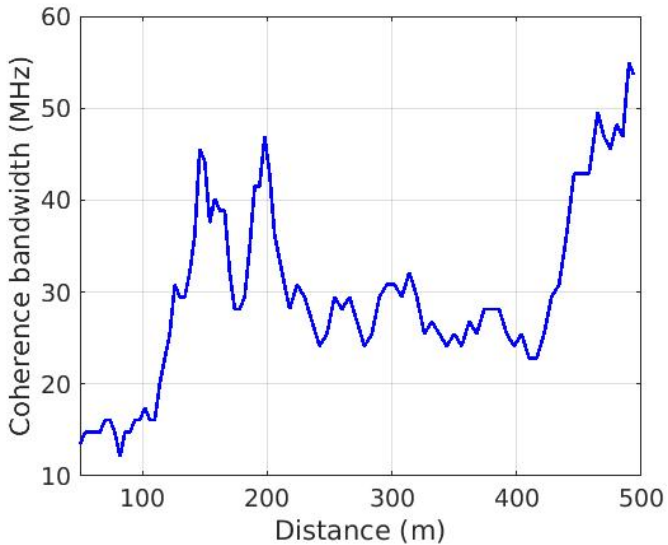

Fig. 3. Coherence bandwidth around a center frequency of 3.4 $\mathrm{GHz}$.

\section{Simulation of the LTE downlink processing}

In LTE [1], the basic modulation scheme is Orthogonal Frequency Division Multiplexing (OFDM) to cope with possible severe frequency selective fading. In the frequency domain, the subcarrier spacing is $15 \mathrm{kHz}$. The preliminary step of the processing is to use a cyclic redundancy check (CRC) by adding a 24-bit checksum to the end of each transport block. However, since CRC has no impact when comparing the performances of various transmission modes, it has not been included in our simulation tool. The code blocks are then sent to a $1 / 3$ turbo coder whose structure is described in [11].

The possible modulation schemes proposed in LTE are QPSK, 16QAM and 64QAM. In the examples given in the next section, only QPSK modulation is used. The MIMO encoder then allows simulating any MIMO architecture as STBC and FSTD and the resulting sequence is formatted into OFDM symbols with 512 subcarriers, among which are 300 data subcarriers.

No cyclic prefix is added in our examples since, whatever the position of Rx in the tunnel, we have seen that the delay spread remains smaller than 20 ns, i.e. much smaller than the duration of an OFDM symbol, equal to $66 \mu \mathrm{s}$. The tunnel thus behaves as an ISI (Inter Symbol Interference) - free channel. Lastly, the received signal is obtained by introducing the measured channel transfer function in the simulation tool and by adding a white Gaussian noise.

\section{Performances of SFBC/FSTD diversity schemes}

In the following, the transmission mode 2, corresponding to a transmit diversity scheme as defined in the LTE release 12 [9] is studied. After recalling the main features of this scheme, BER results along the tunnel are first given without introducing turbo coding. This configuration allows an easiest comparison of the performance of SFBC and FSTD. In a second step, the additional improvement brought by the turbo code is emphasized.

\subsection{Main features of the transmit diversity scheme in LTE}

In the SFBC scheme, diversity is achieved by using $2 \mathrm{Tx}$ antennas. As proposed by Alamouti, if $s 1$ and $s 2$ are two successive symbols, the sequence $[s 1, s 2]$ is sent to the first antenna and the sequence $\left[s 2^{*},-s 1^{*}\right]$ to the second one. Depending on the chosen UE configuration, Rx may have either a single antenna or 2 antennas. These 2 cases will be noted SFBC $(2,1)$ and SFBC $(2,2)$, respectively.

The FSTD scheme uses $4 \mathrm{Tx}$ antennas, pairs of modulation symbols $s 1$ and $s 2, s 3$ and $s 4$, being transmitted by means of SFBC but with transmission alternating between pairs of antenna ports: ports 1 and 3 in one time slot and ports 2 and 4 in the next time slot. This leads to the transmission scheme given in (1) for 4 successive symbols and where the horizontal and vertical lines correspond to time slots and antenna ports, respectively.

$$
\left[\begin{array}{lccc}
s 1 & s 2 & 0 & 0 \\
0 & 0 & s 3 & s 4 \\
-s 2 * & s 1 * & 0 & 0 \\
0 & 0 & -s 4 * & s 3 *
\end{array}\right]
$$

\subsection{Bit error rate without turbo encoder}

The 3 curves plotted in Fig. 4 give the BER for the following configurations: SISO, SFBC $(2,1)$ and SFBC $(2,2)$. The Tx antennas 1 and 10 were chosen (see Fig.1) to have the largest spacing between them $(27 \mathrm{~cm})$. Let us recall that for this QPSK modulation, the SNR is normalized so that its average value at the end of the link, i.e. between 450 and $500 \mathrm{~m}$, is $8 \mathrm{~dB}$.

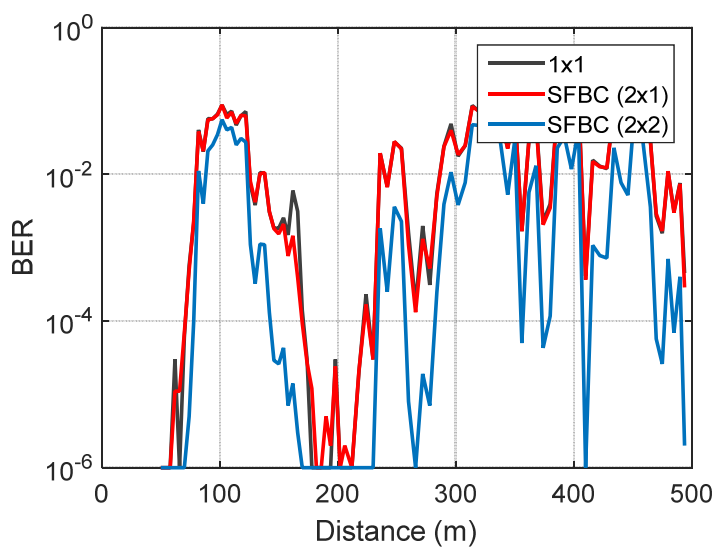

Fig. 4. BER without turbo coding for SISO, $\operatorname{SFBC}(2,1)$ and $\operatorname{SFBC}(2,2)$.

Around $200 \mathrm{~m}, \mathrm{BER}$ is small in any case and this corresponds to a zone of the tunnel where SNR is large, as shown in Fig. 2. If only one antenna is used at the Rx site, improvement brought by using SFBC $(2,1)$ instead of SISO $(1,1)$ remains quite limited, curves in these 2 cases being nearly superimposed. This can be explained by the high correlation between antennas. However, the Alamouti scheme gives better results if 2 antennas are used both at Tx and Rx. 
Curves in Fig. 5 show a comparison between SFBC $(2,1)$ and FSTD $(4,1)$, thus by using an Alamouti approach but based either on 2 or $4 \mathrm{Tx}$ antennas. For FSTD, signal is sent to antennas 1, 4, 7 and 10, to get, once again, the maximum spacing between successive antennas and equal to $9 \mathrm{~cm}$, i.e. one wavelength.

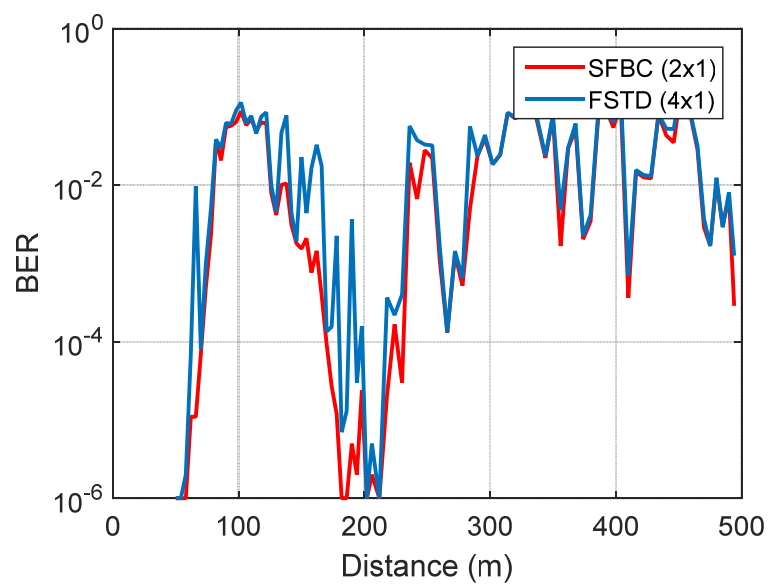

Fig. 5. BER without turbo coding for $\operatorname{SFBC}(2,1)$ and FSTD $(4,1)$.

We see in this Fig. 5 that even with a FSTD scheme, using a single Rx antenna improves BER only slightly.

To be able to easily compare all various configurations, including the case of 1 or $2 \mathrm{Rx}$ antennas, the probability to get a BER smaller than a given value whatever the position of Rx inside the tunnel (at least for the positions where the channel transfer function was measured) is given in Table 2 .

Table 2. Probability to get a BER smaller than a given value, without turbo coding, for SISO and transmit diversity schemes. SNR at the end of the link is assumed to be $8 \mathrm{~dB}$.

\begin{tabular}{|c|c|c|c|}
\hline & $\begin{array}{c}\text { Probability (\%) } \\
\text { BER }<10-2\end{array}$ & $\begin{array}{c}\text { Probability (\%) } \\
\text { BER }<10-3\end{array}$ & $\begin{array}{c}\text { Probability (\%) } \\
\text { BER }<10-4\end{array}$ \\
\hline SISO & 53 & 30 & 22 \\
\hline SFBC $(2,1)$ & 53 & 32 & 22 \\
\hline SFBC $(2,2)$ & 73 & 55 & 44 \\
\hline FSTD $(4,1)$ & 45 & 24 & 14 \\
\hline FSTD $(4,2)$ & 67 & 50 & 38 \\
\hline
\end{tabular}

The comparison of all these BER values show that transmit diversity is interesting only if used in conjunction with $2 \mathrm{Rx}$ antennas to decrease the global correlation between propagation channels. It is important to keep in mind that there is no traffic in the tunnel which could increase the channel diversity and that the total length of the array is rather small, equal to $27 \mathrm{~cm}$.

\subsection{Bit error rate with and without turbo encoder}

Curves in Fig. 6 have been plotted assuming an SFBC $(2,1)$ scheme, BER being calculated with or without turbo coding. Introducing a turbo coding is quite effective since, except in a few zones where SNR $<0 \mathrm{~dB}$ (Fig. 2), BER becomes smaller than $10^{-6}$.

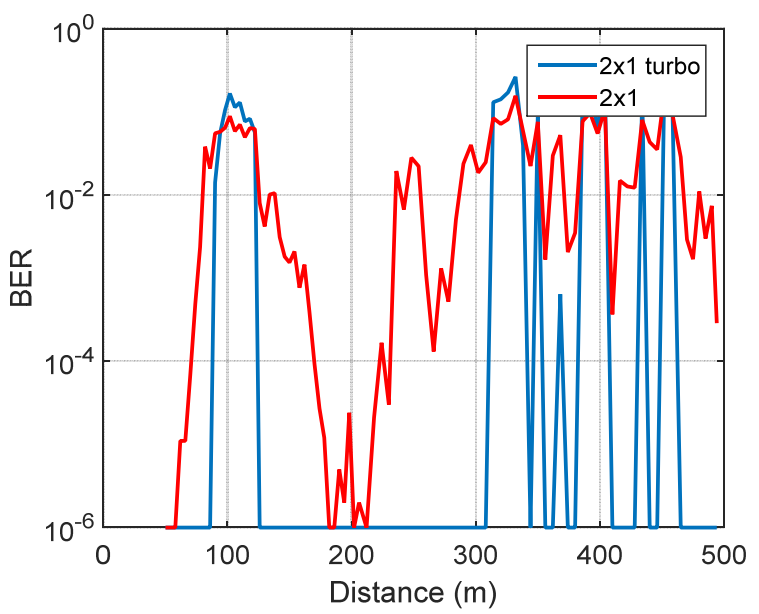

Fig. 6. BER along the tunnel for $\operatorname{SFBC}(2,1)$ without or with turbo coding.

Other measurements are carrying out in tunnels with different cross sections and in presence or not of traffic to see the impact of these parameters on the link performances. The other LTE transmission modes will also be studied.

This work was supported through the OS4 SMARTIES research program by the ELSAT2020 project co-financed by the European Union with the European Regional Development Fund, the French state and the Hauts de France Region.

\section{References}

1. S. Sesia, I. Toufik and M. Baker, Environment LTEThe UMTS long term evolution: From theory to practice (J. Wiley, 2011)

2. H. Holma and A. Toskala (Ed.), LTE advanced: 3GPP solution for IMT-advanced (J. Wiley, 2012)

3. W. B. Yang and M. R. Souryal, NIST report 7986 (2014)

4. S. Malisuwan, J. Sivarak, N. Madan, N. Suriyakrai and P. Promladpanao, J. of Adv. in Computer Networks, 2, 178-181 (2014)

5. P. Kyritsi and D.C. Cox, Electron. Let., 38, 10561057 (2002)

6. C. Garcia Pardo, J. M. Molina-Garcia-Pardo, M. Lienard, D. P. Gaillot and P. Degauque, PIERS M, 22, 91-107 (2012)

7. J. A. Valdesuerio, B. Izquierdo and J. Romeu, IEEE AWPL, 9, 1099-1102 (2010)

8. M. Lienard, C. Sanchis-Borras, J. M. MolinaGarcia-Pardo, D. P. Gaillot, P. Laly and P. Degauque, IEEE AWPL, 13, 122-125 (2014)

9. http://www.3gpp.org/specifications, (2012)

10. A. F. Molish, Wireless communications (J. Wiley 2005)

11. H. Zarrinkoub, Understanding LTE with MATLAB: From Mathematical Modeling to Simulation and Prototyping (J. Wiley 2014) 